\title{
El trabajo de hogar y de los cuidados como nicho de empleo para las mujeres inmigrantes. El caso de ecuatorianas en Zaragoza.
}

\author{
Ana Lucía Hernández Cordero \\ Departamento de Psicología y Sociología, Facultad de \\ Ciencias Sociales y del Trabajo. Universidad de Zaragoza. \\ acordero@unizar.es \\ Viviana Paola Paredes Morales \\ Universidad Nacional de Educación a Distancia. \\ paolaviviana94@gmail.com \\ Elena Luminita Tanase \\ UNIVERSIDAD DE ZARAGOZA. \\ elenaluminita19@gmail.com
}

Resumen: Muchos de los inmigrantes que han llegado a España durante los últimos veinte años, se han insertado en un mercado laboral cada vez más precario y estratificado según etnia y sexo, especialmente como mano de obra a bajo coste en los sectores de la construcción y en el sector servicios de baja cualificación. En el artículo destacamos el caso de las mujeres latinoamericanas que 
trabajan en el ámbito de los cuidados y del trabajo doméstico, para conocer sus vivencias y sus vulnerabilidades ligadas a estos tipos de empleo. Por ello, hacemos visibles las condiciones laborales que viven las inmigrantes que se dedican al empleo de hogar en la ciudad de Zaragoza. Gracias a diez entrevistas en profundidad a mujeres ecuatorianas que han trabajado o que siguen trabajando en este sector, describimos las dinámicas en las que ellas participan en el contexto del país de destino, las dificultades que han tenido en los últimos años de crisis económica, así como los cambios experimentados con la puesta en vigor del Real Decreto 1620/2011 por el que se regula la relación laboral de carácter especial del servicio del hogar familiar.

Palabras Claves: Feminización de la migración, crisis de los cuidados, empleo de hogar, crisis económica, trabajo doméstico y de los cuidados, mujeres inmigrantes ecuatorianas.

\section{The housework and care as an employment for immigrant women.}

\section{The case of Ecuatorian women in Zaragoza.}

Abstract: Many immigrants that have arrived in Spain over the last 20 years have been inserted into a labour market that it is increasingly precarious and is stratified by ethnicity and sex, especially for those in the lowwage workforce in the construction and low qualified service sectors. In this article, we highlight the case of Latin-American women in the care-taking field and that of domestic work in order to explore their life lessons and vulnerabilities bound to this type of work. With this aim, we shed light on the labour conditions of immigrants who dedicate themselves to home care in the city of Zaragoza. Based on 10 in-depth interviews with Ecuadorian women who have worked or are still working in this sector, we present the dynamics in which they participate within the context of the country of destination, the difficulties that they have had in the recent years of economic crises as well as the changes faced with the enactment of Royal Decree 1620/2011 on 14 November by which home care workers are regulated.

Keywords: Feminization of migration, crisis of care, domestic work, economic crisis, housework and care, ecuatorian inmigrant women. 


\section{El trabajo de hogar y de los cuidados como nicho de empleo para las mujeres inmigrantes. El caso de ecuatorianas en Zaragoza.}

Ana Lucía Hernández Cordero

Viviana Paola Paredes Morales

Elena Luminita Tanase

Recibido: 28/02/2017

Aceptado: 14/06/2017

Las mujeres inmigrantes, están siendo empleadas para sustituir el cuidado no remunerado, en un mercado privado y sin regulación, precario y escasamente profesionalizado. Las trabajadoras del hogar han pasado a desempeñar un rol esencial, no solo en la participación laboral de las mujeres autóctonas y el funcionamiento de las familias, sino como elemento de soporte y garantía de la sostenibilidad del Régimen de Bienestar en España (SEDOAC, Día Internacional de las Trabajadoras del Hogar, parr.2, 20 de Marzo de 2014).

\section{INTRODUCCIÓN}

Existen múltiples razones, tanto en origen como en destino, que impulsan a la población extranjera a tomar la decisión de inmigrar hacia España. En el caso de Latinoamérica, destacan la precaria situación de conflicto en el ámbito político, económi$\mathrm{co}$, social y cultural que atraviesan muchos países latinoamericanos como consecuencia de la crisis de los años ochenta, unido a la búsqueda de un futuro mejor (Zúñiga García-Falces, 2005). Asimismo, las transformaciones acontecidas en España en los 
últimos años, tales como el desarrollo económico y social, con la consolidación del Estado de Bienestar y el aumento del nivel educativo y de la calidad de vida de la mayor parte de su población, han significado un crecimiento de las expectativas de la población autóctona a la hora de insertarse al mercado laboral (Cachón Rodríguez, 2002).

La crisis empezada en 2008 supuso un deterioro en los diferentes pilares del bienestar en España. Sin embargo, este periodo de inestabilidad no provocó la salida inmediata de la población extranjera ${ }^{1}$. Una de las consecuencias más acusantes de esta crisis económica, fue la consolidación de un mercado global flexible, caracterizado por el desempeño de trabajos precarios que no todo el mundo está dispuesto a realizar, recayendo así en las personas que tienen una menor capacidad de negociación contractual, como la población inmigrante (Standing, 2011).

El hecho de que siga habiendo personas que migren hacia España se debe, entre otras razones, a que incluso en esta época crítica, el mercado laboral español sigue demandando trabajadores extranjeros, porque existen ocupaciones que no son cubiertas por la población autóctona (Cachón Rodríguez, 2009).

En la actualidad además, se observa una sensible y progresiva feminización de las migraciones, es decir, un fenómeno que se sustancia en el aumento cualitativo y cuantitativo de las mujeres migrantes. Según datos del INE, el porcentaje de mujeres provenientes de países latinoamericanos en 2015 (57,04\%) superaba al de los hombres (42,95\%). Esta feminización está íntimamente relacionada con la demanda de mano de obra femenina para trabajar en el empleo de hogar y de los cuidados (Pérez Orozco y López Gil, 2011). Esto se debe a varios factores como por ejemplo la mayor participación de las mujeres autóctonas en el mercado de trabajo, la escasa incorporación de los hombres a la esfera reproductiva, el progresivo envejecimiento de la población española, la facilidad a la hora de tener una empleada de hogar debido a la escasa regularización de esta actividad y las dificultades para poder conciliar vida familiar y profesional, debido al poco desarrollo de los servicios públicos para cubrir estas necesidades (Parella, 2003). Todo ello, ha llevado a un desajuste

${ }^{1}$ Es a partir del 2012 que se empieza a notar un descenso en el número de extranjeros residentes en España (Hernández, 2015). 
entre las necesidades de cuidados y las posibilidades de proporcionarlos, situación denominada como "crisis de los cuidados" (Pérez Orozco, 2006) caracterizada por la externalización y mercantilización del trabajo de cuidados, que anteriormente era desempeñado gratuitamente por las mujeres dentro de sus hogares.

En España, el empleo de hogar y de los cuidados cuenta con un total de 176.410 mujeres extranjeras afiliadas al Régimen Especial de Empleadas de Hogar, número que representa el 93,13\% del total de la población extranjera afiliada a este régimen (Ministerio de Empleo y Seguridad Social, 2016). La elevada presencia de inmigrantes que trabajan en el sector del empleo de hogar y de los cuidados demuestra por un lado, que existe una demanda importante que estas mujeres están cubriendo, y por el otro, que es su puerta de entrada al mercado laboral español. De hecho, se puede decir que prácticamente es la única opción laboral que éstas encuentran cuando llegan a España, independientemente de su formación y experiencia laboral previa (Parella, 2003). Además, constituye una de las principales vías a la hora de regularizar su estatus de residentes legales en el país de destino (Rodríguez Martínez et al., 2008).

Estudiar la organización social de los cuidados es una temática relevante para el análisis actualizado y en profundidad de nuestra sociedad. En particular, nos interesa enriquecer el debate con información de mujeres ecuatorianas en Zaragoza, por tres razones: en primer lugar, porque los flujos migratorios ecuatorianos desde 1998 y 2004 se han feminizado (Pedone, 2006), y aunque en la actualidad la migración ecuatoriana ha disminuido, las mujeres de ese país siguen teniendo una presencia fuerte en España; en segundo lugar, porque este colectivo ha tenido gran relevancia en diversos estudios realizados a nivel nacional, por ejemplo destacamos a Pedone (2006), Herrera (2007 y 2011) y Camacho (2010). Sin embargo, no existe hasta el momento nada escrito sobre mujeres ecuatorianas en Zaragoza, a pesar de que Ecuador ocupa el cuarto lugar de extranjeros en Zaragoza, con $6.29 \%$, después de Rumanía, Marruecos y Nicaragua (Padrón Municipal, 2014). Por tanto, nuestro trabajo puede considerarse pionero al explorar un fenómeno relativamente nuevo y poco conocido en profundidad. En tercer lugar, porque según la literatura revisada, el sector de los cuidados es donde la mayoría de 
población femenina inmigrante se inserta laboralmente (Cachón, 2002; Parella, 2003).

Por lo anterior, en este artículo nos interesa poner en evidencia las condiciones laborales de las mujeres ecuatorianas que trabajan en el empleo de hogar y de los cuidados en la ciudad de Zaragoza, reflexionar sobre el impacto de la crisis económica en dichas condiciones y los efectos surgidos a partir de la implantación del Decreto 1620/2011 de 14 de noviembre, por el que se regula la relación laboral de carácter especial del servicio del hogar familiar, resaltando las vivencias y vulnerabilidades que suponen este tipo de empleo.

\section{MIGRACIONES INTERNACIONALES Y FEMINIZACIÓN DE LOS FLUJOS MIGRATORIOS}

El aumento de la migración no puede explicarse únicamente como consecuencia de los efectos de "expulsión" de los países de origen y de "atracción" de los países receptores, sino que también hay que entender estos flujos dentro del sistema social, económico y político mundial. La globalización, y la liberalización económica, que promueven la competencia y el individualismo, junto con el desarrollo tecnológico, que favorece la comunicación instantánea, son otros de los elementos imprescindibles para entender la dinámica de las migraciones internacionales (Camacho, 2010).

La llegada de la globalización supuso la aceleración de los flujos de dinero, información, bienes y servicios, marcados por relaciones de poder que acentúan las desigualdades entre los países ricos y los países pobres. La re-estructuración social y económica generada por la globalización y el capitalismo liberal ha influido en el aumento de los movimientos migratorios, además de un cambio sustancial de sus características constitutivas (Gil Araújo, 2005). Uno de los cambios más importantes y reciente ha sido la feminización de los flujos migratorios (Herrera, 2011).

La economía de los servicios, a partir de los años ochenta, redujo la demanda de trabajadores masculinos al tiempo que aumentó la demanda de mano de obra femenina y barata para trabajar en este sector. La terciarización de las economías de los países occidentales más avanzados, particularmente la expansión 
del mercado laboral para servicios personales, creó una demanda específica de mano de obra femenina (Pérez Orozco et al., 2008).

En el caso de Ecuador, la migración internacional hacia Europa de la población adulta se produjo precisamente por la crisis política y socioeconómica en los años ochenta, que supuso la aplicación de políticas de ajuste estructural sustentadas en el neoliberalismo salvaje y en el aumento de la corrupción en este país latinoamericano (Pedone, 2006). La menor posibilidad de las mujeres ecuatorianas de acceder al mercado laboral remunerado en su país les impulsó a buscar mejores oportunidades laborales en otros países (Camacho, 2010).

Por su parte, en los países de destino como es España, tienen lugar elementos de atracción que influyen en la decisión de migrar (Cachón, 2002). Su mercado de trabajo, por ejemplo, presenta una aguda estratificación laboral en función del origen étnico, el estatus migratorio y el género. Como consecuencia de esta estratificación, el conjunto de la población inmigrante se ve abocada a las ocupaciones de menor estatus social, menor remuneración y peores condiciones laborales, ocupando las mujeres inmigrantes el último escalón: el empleo de hogar y de los cuidados (Parella, 2003).

En el caso español, la principal razón por la que las ecuatorianas han elegido España como destino migratorio se relaciona estrechamente con una demanda de mano de obra que cubra los puestos de trabajo en el mercado de los cuidados. Esto a su vez tiene que ver con elementos puntuales. Por ejemplo, una política migratoria que ha favorecido la entrega de permisos laborales para el empleo de hogar y de los cuidados sobre otras ocupaciones (Hernández Cordero, 2015).

\section{EMPLEO DE HOGAR Y CUIDADOS COMO NICHO LABORAL DE LAS MUJERES INMIGRANTES}

En el presente artículo, al hablar de empleo de hogar y de los cuidados, nos referimos a una modalidad del servicio de proximidad, que se caracteriza por prestar servicios a personas particulares, no a empresas, a cambio de una retribución. Dicha ocupación engloba tanto las tareas de mantenimiento de la infraestructura de la casa como los cuidados de los miembros de la 
familia empleadora: niños/as, personas mayores y/o dependientes. Es posible distinguir tres tipos de contratación en función del tiempo de presencia en el hogar empleador, las condiciones laborales y la intensidad del vínculo con los empleadores (Parella, 2003).

\section{Modalidad en régimen interno.}

Esta modalidad se caracteriza porque la empleada reside en el hogar donde desempeña su trabajo. Aunque existen periodos de descanso pactados entre la empleada y el empleador, el propio hecho de residir en el lugar de trabajo lleva implícita una disponibilidad prácticamente del $100 \%$, según sean las necesidades de la familia empleadora, lo que genera largas jornadas de trabajo. Sumado a esta situación, las mujeres empleadas, por el hecho de vivir en la casa empleadora, ven reducida en gran medida su privacidad.

\section{Modalidad en régimen externo.}

En esta modalidad se identifican dos variantes: la primera denominada como empleo externo fijo, en el cual la empleada trabaja a jornada completa para una única familia empleadora sin residir en su domicilio, lo que permite un mayor respeto a los horarios establecidos y una mayor privacidad y autonomía. Con frecuencia, el paso de interna a externa corresponde a la obtención de las primeras tarjetas de residencia (Pérez Orozco, 2011; Herrera, 2011); la segunda se trata del empleo externo por horas, que consiste en trabajar a tiempo parcial en uno o varios hogares, dentro de un horario definido. Es habitual que las empleadas acumulen la cantidad de casas y horarios que les es posible atender, en muchas ocasiones esto supone quedarse en una zona o barrio para evitar "perder tiempo" en la movilidad entre un hogar y otro.

El empleo de hogar es un trabajo que tiene muy poca consideración social y bajo reconocimiento institucional, como consecuencia de la devaluación que sufren los trabajos "típicamente feminizados" (Andall, 2000) y que se llevan a cabo en los espacios privados de los hogares. Estas características la hacen una ocupación más feminizada y que concentra el número más alto 
de inmigrantes en España: más del 90\% son mujeres y, de ellas, el $60 \%$ son inmigrantes (EPA, 2015).

Aunque este tipo de trabajo no goza de especial relevancia a nivel político, en 2011 se dan dos cambios normativos muy importantes para este sector: La Ley 27/2011 sobre Actualización, Adecuación y Modernización del Sistema de Seguridad Social que incorporó el antiguo régimen especial del empleo del hogar al régimen general; y el Real Decreto 1620/2011 por el que se regula la relación laboral de carácter especial del servicio del hogar familiar que revocó la legislación existente desde 1985. Estos cambios han supuesto unos avances en materia de la reglamentación del empleo de hogar al mismo tiempo que han influido en las condiciones de las empleadas de hogar y de los cuidados (Goñalons-Pons y Hernández Cordero, 2013).

Estas reformas definen un nuevo marco normativo sobre la relación laboral entre empleada y empleador. El Real Decreto de 1985 definía la relación laboral del empleo de hogar y de los cuidados como "especial", por el hecho de llevarse a cabo dentro de un hogar particular y basarse en una relación de confianza. Esto dificultaba su profesionalidad, el reconocimiento de sus derechos e invisibilizaba las condiciones laborales, es decir, no favorecía su transparencia y su posible control legal (Goñalons-Pons y Hernández Cordero, 2013). Como señalamos anteriormente, tanto la Ley 27/2011 como el Decreto 1620/2011 modifican esta definición de "especial" y se empieza a entender el empleo de hogar dentro del régimen general de la Seguridad Social.

Es a partir de este cambio que tienen lugar dos modificaciones beneficiosas para las empleadas de hogar. En primer lugar, logra establecerse un salario mínimo por trabajar a jornada completa igual al Salario Mínimo Interprofesional (SMI), y además tienen derecho a las dos pagas extraordinarias que no pueden ser inferiores al SMI en cómputo anual. En segundo lugar, los empleadores pasan a tener la obligación de registrar a la empleada en la Seguridad Social, de informarle sobre las condiciones laborales y los elementos esenciales de su contratación y de formalizar así el contrato por escrito. A pesar de estas modificaciones, el cambio normativo no han supuesto una real equiparación y el empleo de hogar sigue teniendo un carácter especial dentro del régimen general. 
En lo que se refiere a la extinción del trabajo, el Real Decreto de 1985, establecía que las empleadas de hogar no tenían derecho a desempleo y que se podría llevar a cabo un despido por desistimiento, es decir, no alegando causas objetivas. Con la reforma legislativa del 2011, esto no ha cambiado, colocando a las empleadas en una situación de mayor vulnerabilidad e incertidumbre.

Otro aspecto a destacar es la imposibilidad de ser empleada del hogar discontinua. Esto provoca una relación de subordinación con respecto al empleador ya que depende de éste a la hora de cotizar y acceder a los derechos. Esta modificación repercute en mayor medida en la población extranjera, ya que dificulta la renovación de los permisos de residencia aumentando el trabajo informal y con ello, las condiciones laborales precarias (Goñalons-Pons y Hernández Cordero, 2013).

Asimismo, el acceso a la justicia depende de la inspección de trabajo y el acceso a canales que permitan denunciar abusos e incumplimientos. En cuanto a la inspección laboral, el Real Decreto de 1985 imposibilitaba el acceso al hogar del empleador sin consentimiento de este, salvo que existiera una denuncia. Con la nueva reforma, este aspecto continúa vigente ya que se prioriza la privacidad del hogar empleador frente a los derechos de las trabajadoras. En cuanto a los mecanismos de denuncia, estos siguen siendo poco utilizados por las empleadas debido al poco acceso a la información que tienen, que en el caso de las internas, se agudiza debido al aislamiento, la falta de tiempo y el bajo nivel de sindicalización.

Para finalizar, a raíz de las diferencias observadas, podemos decir que estas modificaciones normativas no han mejorado de manera significativa las condiciones de trabajo de las empleadas de hogar y de los cuidados. Esto significa, que la legislación vigente no es suficiente. Al respecto destacamos que es sumamente necesario que España ratifique el Convenio 189 sobre trabajo decente para las trabajadoras y trabajadores domésticos de la Organización Internacional del Trabajo (OIT) de junio de 2011, con el objetivo de dignificar y, principalmente, equiparar 
las condiciones de trabajo del sector del empleo de hogar al resto de sectores ${ }^{2}$.

\section{APUNTES METODOLÓGICOS}

A partir del marco normativo antes descrito, en este artículo se recogen parte de los resultados obtenidos en la investigación realizada durante el año 2015. Con base en una metodología cualitativa, en este estudio nos importaba conocer y profundizar en la situación que viven las empleadas de hogar a partir de sus relatos y sus puntos de vista, yendo más allá de los aspectos externos y de la mera cuantificación de los datos. Para tal propósito, hemos elegido llevar a cabo entrevistas semiestructuradas que recopilan estas informaciones. En cada una de las entrevistas se ha hecho especial hincapié en los efectos de la crisis económica en sus ámbitos laborales y en su percepción con respecto a los cambios normativos del empleo de hogar.

Nos hemos preocupado por contar con mujeres que hayan trabajado de forma continua, con o sin contrato, durante el periodo comprendido entre 2007 y 2009 , y mujeres que hayan trabajado con contrato laboral, al menos durante los años comprendidos entre 2010 y 2012, para contar con relatos que nos permitieran interpretar sus historias en dos momentos distintos de la crisis, es decir, justo cuando la inestabilidad laboral alcanzó su nivel más alto en España. Así, a través de la técnica de la Bola de Nieve hemos podido contactar a diez mujeres de nacionalidad ecuatoriana con edades comprendidas entre 30 y 55 años que cumplen con las características anteriormente señaladas.

Las entrevistas constaron de una única sesión en la que se siguieron los pasos necesarios para garantizar una conducta ética propia de la investigación social: en primer lugar, se les explicó el objetivo del estudio y los temas que se iban a tratar, y posteriormente se les informó que toda la información recogida era confidencial, anónima y voluntaria. Al finalizar cada entrevista

${ }^{2}$ Al respecto, es importante apuntar que durante el I Congreso de Empleo de Hogar y de Cuidados realizado en Madrid el 1 y 2 de Octubre de 2016 y organizado por el Grupo Turín y el Ayuntamiento de Madrid, se señaló con contundencia que el reconocimiento social y político del empleo de hogar pasa por la ratificación del Convenio 189 de la OIT. 
se les ofrecieron datos de contacto para cualquier eventualidad posterior.

\section{RELATOS DE ECUATORIANAS TRABAJADORAS DEL HOGAR: CONDICIONES LABORALES EN LA ENCRUCIJADA DE LA CRISIS}

Las mujeres entrevistadas han llegado a España entre 19992001, coincidiendo con la feminización de los flujos migratorios ecuatorianos (Pedone, 2006). Se trata de mujeres que han migrado individualmente y muchas de ellas se erigen como pioneras de los flujos migratorios.

Como consecuencia de la crisis que estaba atravesando Ecuador, a principios del Siglo XXI (Herrera, 2007), y la demanda para trabajar en el empleo de hogar y de los cuidados en España (Parella, 2003), la migración se convirtió en la opción más viable. Todas las mujeres entrevistadas contaron con la ayuda de familiares y amigos, tanto a la hora de emigrar como a la hora de encontrar trabajo en España. De hecho, algunas venían ya con ofertas de empleo cerradas y tardaron uno o dos días en empezar a trabajar.

"Porque una compañera de ahí mismo de Loja que trabajamos juntas y ella se había venido antes y no me había avisado. Entonces cuando ella estuvo aquí me dijo y me llamo, oye te quieres venir... bueno pues dije me voy, pero así fue de un momento a otro, no fue nada pensado... bueno le dije bueno pues que me voy y me dijo te voy a tener trabajo ya aquí para que vengas, y vine a cuidar a una niña" (Marianita $^{3}$, 18 años en España, 9 años trabajando como empleada de hogar).

En cuanto al tiempo de estancia, todas nuestras participantes llegaron con la intención de quedarse un tiempo relativamente corto y luego regresar a su país de origen. Sus planes consistían, principalmente, en obtener dinero suficiente para desarrollar sus proyectos en Ecuador (construir una casa, abrir una farmacia, etc.). No obstante, una vez que llegaron a Zaragoza, los planes fueron cambiando al punto que decidieron reagrupar a sus hijos/ as y esposos.

${ }^{3}$ Todos los nombres que aparecen en los testimonios son seudónimos. 
La razón de este cambio de planes se debe, en primer lugar, a que desconocían por completo todo el sistema migratorio español y las dinámicas propias del mercado laboral. En segundo, porque las condiciones en Ecuador y familiares no resultaron ser las adecuadas, por ejemplo, enviaron dinero y éste no fue destinado a los proyectos planificados, o no pudieron disponer de él, debido al congelamiento de los bancos en Ecuador.

A continuación, presentamos las condiciones en las que las mujeres entrevistadas han desempeñado su trabajo, en años particularmente problemáticos para el mercado de trabajo en su país de destino.

En primer lugar, señalamos la relación entre las condiciones laborales y el estatus migratorio, porque ayudará a entender la situación de las mujeres entrevistadas. Según la normativa española, que regula la entrada y el asentamiento de las personas extranjeras, existe una relación directa entre contrato de trabajo y la tarjeta de residencia, que marca básicamente que sin contrato de trabajo no pueden solicitar la tarjeta de residencia, con algunas excepciones como reagrupaciones familiares, familiar de comunitarios y razones humanitarias. El sistema establece que los extranjeros no comunitarios tienen que renovar su permiso de residencia anualmente los primeros dos años, para obtener posteriormente una tarjeta para cinco años y una más para diez años.

Ocho de nuestras diez entrevistadas han trabajado con contrato, independientemente de su modalidad específica de empleo, pero en todos estos casos el contrato ha llegado después de un periodo más o menos extenso (años) de trabajo, sin ninguna formalización de la relación laboral. Los períodos en los que han realizado sus tareas sin contrato, lo han hecho principalmente por no contar con la tarjeta de residencia que supone el permiso de trabajo.

La modalidad de empleo de hogar a la que nuestras participantes han podido tener acceso, ha variado en función de la tenencia o no de su familia en España, de la pertenencia de la residencia legal y del tiempo que llevan residiendo. De esta cuenta, al principio de su estadía en territorio español, sin familia, y sin la autorización para trabajar, han estado siempre en la modalidad de internas. Al contrario de lo que ocurre cuando regularizan su situación, después de varios años de residir en España, y consiguen iniciar los 
tramites de reagrupación. Según los testimonios, estas condiciones las empujan a buscar trabajo en la modalidad externa, como fija o por horas. Así lo relata una entrevistada:

"Al principio fue mejor de interna, era más fácil encontrar trabajo porque habían muchas familias que solo así querían y pues yo sin nadie aquí, me ahorraba pagar a parte el cuarto, el transporte y además me contaron en la iglesia que a veces la policía controla en las salidas de metro, entonces también me ahorraba ese susto. Ya después, con los papeles, si que fue mejor cambiarme de externa, igual que hizo Oralia" (Rosa, 14 años en España y 14 trabajando como empleada de hogar)

El empleo de hogar y de los cuidados implica una cobertura de las necesidades básicas que presentan las personas a lo largo del ciclo vital, imprescindibles para su desarrollo óptimo y necesario para el mantenimiento de la vida (Pérez Orozco, 2006). Se trata por ejemplo de limpieza, cocina, compra de víveres, atención a medicamentos, etc. No obstante, estas tareas no suelen estar especificadas al momento de la contratación, porque tal como señalan las entrevistadas, las familias siempre han dado por hecho lo que implica "el trabajo doméstico" y además entienden que se trata de una labor que por ser mujeres saben de qué trata. Esta poca claridad en las tareas a desempeñar, en ocasiones supone que se vayan sumando responsabilidades que no han sido pactadas, así como horas de trabajo que no son remuneradas.

"Sí, lo que pasa es que cuidando a esa abuela es estar allí con ella, si quiere un vaso de agua, si quiere ir al baño y tal... pero ya después hacía la limpieza también.... después ya me puso a planchar la ropa y cosas así, me traía la ropa de la hija para que se la planche... y tal... Yo estaba de lunes a viernes, los sábados no iba... unas veces me llamaban otras no... pero claro los sábados que iba no me los pagaba... me pagaban de lunes a viernes y allí incluido el sábado" (Ana, 14 años en España, 10 años trabajando como empleada de hogar).

En cuanto al salario, este varía tanto en función de la modalidad de contratación como de la situación administrativa de la empleada. Cuando éstas no cuentan con la tarjeta de residencia, se encuentran en una situación de desventaja para negociar 
tanto condiciones como salario. En estos casos suelen ser los empleadores los que fijan el salario, sin que exista una previa negociación.

La situación cambia una vez que obtienen su permiso correspondiente y un contrato de trabajo. El cambio de normativa ha ayudado mucho a que se respeten los derechos de estas trabajadoras, pues con contrato y cotizaciones a la Seguridad Social, en jornada completa (externa o interna) no pueden recibir menos del SMI. En el caso de nuestras entrevistadas, sus experiencias oscilan entre 600 euros, menos del SMI, y 1000 euros al mes.

Con respecto a las vacaciones, también varían según la modalidad de contratación. Por ejemplo, las internas y externas con jornada completa, tienen un mes de vacaciones, mientras que quienes trabajan por horas, por no cobrar durante el periodo vacacional, se organizan de tal manera que a veces directamente no gozan de ningún período festivo. Lo común es que las vacaciones no suelen estar pactadas desde un principio, sino que tienen lugar en función de los intereses de los empleadores. En algunos casos, será en los periodos vacacionales de las empleadoras, pues no están dispuestas a pagar por un servicio que pueden hacer ellas mismas, por ejemplo, los meses de verano. En otros casos, la situación es inversa: durante el verano se llevan a la empleada para que trabajen y posponen su periodo de descanso a otros meses del año.

"Sí, porque ellos normalmente en Agosto se iban de vacaciones a EE.UU, A Brasil o a Londres... por allá... y como no me podían llevar pues ellos se iban... se iban y me dejaban a mi esos días... me daban fiesta" (Ana, 14 años en España, 10 años trabajando como empleada de hogar, actualmente trabaja en una empresa de embutidos).

“...yo nunca cogía mis vacaciones para mí porque me iba con ellos y me pagaban el doble o sea ganaba 1000 y ese mes me salía por 2000" (Ketty, 13 años en España, 13 años trabajando como empleada de hogar, actualmente trabaja como empleada de hogar).

Con respecto a la relación empleada-empleador, nuestras participantes insisten en la ambigüedad que han vivido y que en pocas ocasiones han logrado superar con facilidad: por un lado, existe una relación contractual en la que se da una venta 
de servicios y una compra de los mismos; pero por el otro, el hecho de tener lugar dentro de los hogares da cabida a un trato "más personal, aparentemente más directo, que va más allá de una mera relación laboral", en la que no se clarifica que se trata de un trabajo como "cualquier otro" con obligaciones y derechos de ambas partes.

“... cuando entre a trabajar en esa casa la señora me dice mira Ana tú vas a trabajar interna aquí y vas a ser un miembro más de la familia... tú no te cortes, no te de pena de nada, porque tú vas a vivir aquí con nosotros y tenemos que convivir , aprender a llevarnos y a entendernos... y que más que una empleada tú vas a ser una más de la familia... y así quiero que nos veas que tengas confianza y tal porque si no... para poder convivir" (Ana, 14 años en España, 11 años trabajando como empleada de hogar, actualmente trabaja en una empresa de embutidos).

Es común escuchar que se habla de la empleada "como si fuera de la familia", debido a la existencia de una relación de confianza mutua. No obstante, esto repercute en que se confunden derechos y obligaciones con favores, tanto por parte de la empleada como de la empleadora. Estamos hablando de no cobrar por las horas extras, dar regalos en lugar de pagar los servicios, cambiar horarios o fechas de vacaciones sin previo aviso, etcétera. Un ejemplo muy usual, que nuestras entrevistadas nos comentan, es que cuando es día libre y ellas se quedan en casa por diferentes motivos, dedican su jornada como cualquier otra: preparando comida, sirviendo, limpiando, etc., sin atreverse a cobrar por esos servicios prestados.

"Entonces la señora le dice encima que te hacemos el favor de contratarte quieres que te paguemos más... algo así le había dicho. Y a ella le hacían ir los fines de semana. Ella entraba a las 17 de la tarde y ya se quedaba hasta el siguiente día, el sábado... y el domingo también" (Sara, 12 años en España, 10 años trabajando como empleada de hogar).

Nuestras entrevistadas hacen hincapié en su experiencia personal y en los relatos de otras compañeras, con las cuales se relacionan, para poder afirmar que el empleo de hogar y cuidados se ha visto afectado con la llegada de la crisis económica en España. 
Según ellas, los mayores efectos negativos se concentran en la bajada de los salarios y la disminución de los puestos de trabajo, principalmente el empleo por horas.

"Sí, sí, mucho.... Es que me bajaron las horas de trabajo cantidad. Uff, trabajaba mucho y después me fueron bajando de 8 a 6 , de 6 a 4, de 4 a 2 y después me dijeron que ya no tenían trabajo para mí. Totalmente me quede sin trabajo. Yo antes tenía muchísimo trabajo." (Lucía, 15 años en España, 10 años trabajando como empleada de hogar, actualmente trabaja en el negocio de su hija).

En el caso de las internas o externas con jornadas fijas, el trabajo está mayormente vinculado al trabajo de cuidados. En este ámbito, la demanda ha seguido aumentando debido al progresivo envejecimiento de la población española.

En cuanto a los cambios en la normativa, es necesario señalar un importante desconocimiento de esta materia por parte de nuestras participantes al estudio. Estas mujeres admiten no conocer mucho o desconocer en la práctica concreta los elementos centrales de la reforma, admitiendo que la suya suele ser una actitud pasiva con respecto a esta problemática. Esto se refleja en el hecho que ellas a menudo se han dejado llevar por lo que sus empleadores les indican como "legal", "dentro de la norma" o, más coloquialmente, limitándose a "así se hacen las cosas". La actitud que muestran todas las entrevistadas es de confianza hacia sus empleadores pero también de resignación a la hora de enfrentarse a todas las cuestiones administrativas y burocráticas relativas a sus empleo, porque efectivamente ellas mismas reconocen una falta de información sobre sus derechos y obligaciones. En ese sentido, se hace evidente un vacío en cuanto al conocimiento de cómo se regula el empleo de hogar en España y cuales son las implicaciones legales para las trabajadoras. Al respecto, es importante señalar que ese vacío se está empezando a cubrir con la creación de la Asociación de Trabajado@s del Hogar y de Cuidados de Zaragoza ${ }^{4}$, iniciativa impulsada por un grupo de empleadas de hogar de varias nacionalidades (latinoamericanas

${ }^{4}$ El 28 de mayo de 2017 se ha llevado a cabo el I Encuentro de Trabajador@s del hogar y de cuidados de Zaragoza con el objetivo de conformarse en una Asociación que forme parte del Grupo Turín y se sume a las actividades de reivindicación de las condiciones laborales del empleo de hogar en España. 
y españolas) que participaron en el I Congreso de Empleo de Hogar y de Cuidados en Octubre de 2016.

A pesar de lo anterior, nuestras entrevistadas han señalado que dentro del poco conocimiento que tienen sobre la Ley, el cambio legislativo les ha perjudicado, específicamente por la imposibilidad de cotizar como profesionales autónomas y de acceder a los subsidios por desempleo.

"No ha habido cambios de nada, con esta ley fue a peor porque antes había personas que se podían cotizar ellas mismas, hacían $\mathrm{x}$ horas le firmaban y le cotizaban y ahora si no tienes quien te firme un contrato no puedes cotizar. Fue a peor para mucha gente. Yo por ejemplo, cuando estaba en el locutorio, algunas les firmaba para reunir horas y poder cotizar y a mí no me perjudicaba en nada, porque claro cómo se pagaban y tal, pero ahora si no tienes un contrato de trabajo y que te lo firmen no puedes darte de alta" (Marianita, 18 años en España, 9 años trabajando como empleada de hogar, actualmente trabaja como empleada de hogar).

"Yo muchos años antes cotizaba pero tampoco tuve beneficio. Imagínate no cobras, lo único bueno es que claro, si te enfermas te siguen pagando, en ese aspecto si pero en otras cosas no, porque no se cobra paro tampoco" (Marcia, 14 años en España, 14 años como empleada de hogar, actualmente trabaja como empleada de hogar).

En su conjunto, el análisis evidencia que la situación de las mujeres ecuatorianas empleadas de hogar y de los cuidados no ha cambiado en esencia sus condiciones laborales. No obstante, podemos afirmar con contundencia, de acuerdo con sus testimonios, que la crisis ha reforzado las precarias condiciones laborales de este sector. Cabe añadir también su queja y desilusión por las nuevas reformas que no han supuesto una mejoría, ni en su condición de trabajadoras ni en su vivencia personal como migrantes.

\section{CONCLUSIONES}

La migración de las mujeres ecuatorianas que se insertan en el ámbito del empleo de hogar y de los cuidados en Zaragoza, coincide con ese flujo feminizado proveniente de Ecuador hacia España. 
Los testimonios recopilados nos muestran que la crisis de los cuidados continua vigente en la ciudad de Zaragoza, principalmente porque las instituciones de gobierno siguen delegando estas tareas a las familias, quienes contratan a mujeres inmigrantes porque no logran cubrir tales necesidades de mantenimiento y reproducción por su cuenta, como el caso de nuestras entrevistadas ecuatorianas. Esto significa, que las mujeres inmigrantes están resolviendo dicha crisis (Pérez Orozco y López Gil, 2011 y Parella Rubio, 2003), y que con frecuencia esta dedicación se realiza a costa de su propio bienestar personal y profesional.

El nicho laboral que demanda mano de obra femenina y migrante, como es el del cuidado, es a la vez muy feminizado y precario, y además carece de una valoración social y normativa que sea positiva y plenamente favorable. La llegada de la crisis económica a España, ha generando unas condiciones laborales aún más inestables en términos salariales y de las efectivas condiciones de trabajo (tareas requeridas) y de dedicación (horarios abusivos y complicada organización de los días libres). La normativa en materia de extranjería sitúa a la población no comunitaria (como en el caso de nuestras entrevistadas) en una situación de vulnerabilidad en lo que se refiere al mercado de trabajo. En el caso de las mujeres que se dedican al empleo de hogar y de los cuidados, la necesidad de mantener sus contratos de trabajo para así poder renovar sus permisos de residencia por ejemplo, les obliga a aceptar condiciones laborales precarias. Esto se debe también al escaso poder de negociación a su disposición, que se hace prácticamente nulo en el caso de aquellas mujeres que no cuentan con el permiso de residencia.

El Régimen Especial a Régimen General y la implantación del Real Decreto 1620/2011 no ha supuesto todos los cambios positivos que pretendía ofrecer, principalmente porque sigue teniendo un carácter "especial" dentro del Régimen General que es poco efectivo ${ }^{5}$. Tal como se encuentra regulado en la actualidad, el empleo de hogar sigue careciendo de esa consideración de un "trabajo como cualquier otro", de manera que las empleadas en este sector, como nuestras entrevistadas, quedan expuestas a condiciones laborales ambiguas, escasamente equilibradas (por

${ }^{5}$ Véase Díaz Gorfinkel y Fernández López, 2016. 
lo que concierne a la relación con el empleador y con las tareas encomendadas) además de fuertemente desprotegidas.

\section{BIBLIOGRAFÍA}

Andall, J. (2000). Gender, Migration and Domestic Service. Idershot: Ashgate.

Atienza Azcona, J. (2005). "La crisis del desarrollo y las migraciones”. En Zúñiga García-Falces, N. (coord.) La migración un camino entre el desarrollo y la cooperación. (pp.53-74). Madrid: Centro de Investigaciones para la Paz.

Cachón, L. (2002). "La formación de la España inmigrante mercado y ciudadanía". Revista Española de Investigaciones Sociológicas 97, 95-126.

Camacho Z, G. (2010). Mujeres migrantes: trayectoria laboral y perspectiva de desarrollo humano. Argentina: CLACSO.

Castelló Santamaria, L. (2008). "La mercantilización y mundialización del trabajo reproductivo. El caso español”. XI Jornadas de Economía Critica. Barcelona: Universidad Autónoma de Barcelona.

Díaz Gorfinkel, M. y Fernández, C. (2016). Impacto de las reforams legislativas en el sector del empleo del hogar en España. Ginebra: OIT

England, P. (2010). "The Gender Revolution: uneven and Stalled”. Gender \& Society, 24(2), 149-166.

Gil Araújo, S. (2005). "Cartografías migratorias: migraciones internacionales en el marco de las relaciones Norte-Sur". En N. Zúñiga García-Calces (coord.), La migración, un camino entre el desarrolo y la cooperación (pp.13-53). Madrid: Centro de Investigación para la Paz

Goñalons-Pons, P. y Hernández Cordero, A. (2013). “¿Reforma para un trabajo digno? Luces y sombra de la reforma del empleo del hogar en España en clave de género y migración". En Actas Oficiales del IV Congreso de la Red Española de Política Social (REPS). Las políticas sociales entre crisis y post-crisis. Universidad de Alcalá.

Gregorio Gil, C. (1998). Migración femenina: su impacto en las relaciones de género. Madrid: Narcea. 
Hernández Cordero, A. (2015). "Cuidados que cruzan fronteras: la colectivización de la maternidad en un contexto migratorio". Revista Acciones e Investigaciones Sociales 35, 89-114.

Herrera, G. (2007). "Ecuatorianos/as en Europa: de la vertiginosa salida a la construcción de espacios transnacionales". En I. Yépez del Castillo y G. Herrera, Nuevas migraciones latinoamericanas a Europa: balances y desafíos (pp.189-216). Quito: Risper Graf C.A.

Herrera, G. (2011). "Cuidados globalizados y desigualdad social. Reflexiones sobre la feminización de la migración andina". Nueva sociedad 233, 87-97.

Hondageno-Sotelo, P. (2011). Doméstica: trabajadoras inmigrantes a cargo de la limpieza y el cuidado a la sombra de la ambudancia. México: Instituto Nacional de Migración.

Ortner, S.B. (1979). “¿Es la mujer con respecto al hombre lo que la naturaleza con respecto a la cultura?", En O. Harris y K. Young, Antropología y feminismo (pp.109-131). Barcelona: Anagrama.

Parella Rubio, S. (2003). Mujer, inmigrante y trabajadora: la triple discriminación. Barcelona: Anthropos.

Pedone, C. (2006). Estrategias migratorias y poder: tú siempre jalas a los tuyos. Quito: ABYA-YALA.

Pérez Orozco, A. (2006). Perspectivas feministas en torno a la economía: el caso de los cuidados. Madrid: Consejo Económico y Social (CES).

Pérez Orozco, A., Paiewonsky, D. y García Domínguez, M. (2008). Cruzando Fronteras: Migración y Desarrollo desde una perspectiva de género. Madrid: Instituto de la Mujer.

Pérez Orozco, A., y López Gil, S. (2011). Desigualdades a flor de piel: cadenas globales de cuidados. Concreciones en el empleo de hogar y políticas públicas. Madrid: Agencia Española de Cooperación Internacional.

Rodríguez Martínez, P., Martín Palomo, M., Holgado Fernández, I., Ruíz Castillo, M., Gorroño Arregui, I., Castilla, E. y Rodríguez Avila, N. (2008). Mujeres, trabajos y empleos: en tiempos de globalización. Barcelona: Icaria.

Servicio Doméstico Activo (SEDOAC). (20 de Marzo del 2014). Día internacional de las trabajadoras del hogar. Recuperado 
de: http://serviciodomesticoactivo.blogspot.com.es/2014/03/ dia-internacional-de-las-trabajadoras.html

Solé, C. (1995). Discriminación racial en el mercado de trabajo. Madrid: Consejo Económico y Social (CES).

Standing, G. (2011). El precariado: una nueva clase social. Barcelona: Pasado y Presente. 